\title{
Prevalence of vitamin D deficiency and insufficiency in Malaysian infants
}

\begin{abstract}
Dear Editor,
Vitamin D deficiency, a worldwide health problem, is also prevalent in tropical countries. ${ }^{1}$ It is estimated that $15 \%$ of the world's population are either vitamin D deficient or insufficient. ${ }^{1}$ In a study on the state of Kelantan in Malaysia (2010-2012), 60\% of pregnant women were vitamin D deficient. ${ }^{2}$ Maternal vitamin D deficiency predisposes infants to vitamin D deficiency especially if they are exclusively breastfed. The prevalence of vitamin D deficiency in Malaysian infants is unknown. We conducted a cross-sectional study to ascertain the prevalence and risk factors predisposing to vitamin D deficiency in Malaysian neonates.
\end{abstract}

We recruited term neonates (gestational age $\geq 37$ weeks; aged 2 to 4 weeks) followed up for neonatal jaundice in a well-baby clinic from December 2016 to December 2017. Written informed consent was obtained from parents. Ethical approval was obtained (Ethics Number: 20161115-4600). No blood samples were obtained purely for the purpose of the study. We excluded neonates with the following: prematurity (gestational age $<37$ weeks), neonatal cholestasis (conjugated bilirubin $>17 \mathrm{mmol} / \mathrm{L}$ if total bilirubin $<85 \mathrm{mmol} / \mathrm{L}$, or conjugated bilirubin $>20 \%$ if total bilirubin $>85 \mathrm{mmol} / \mathrm{L}$ ), infants who were on diuretics or vitamin D supplements, and infants with feeding difficulties. Infants whose mothers had renal, liver, thyroid and gastrointestinal disorders or taking antiepileptic medications were excluded.

We collected data on age, gender, ethnicity, gestational and feeding history. Daily sun exposure of the infants was determined via a set of sunshine exposure behaviour in a questionnaire adapted from Barger and Heaney. ${ }^{3}$ Blood was collected for liver function, serum 25-hydroxy vitamin D $(25(\mathrm{OH}) \mathrm{D})$ and bone profile tests. Serum 25(OH)D level was assayed using Siemens Advia Centaur Analyser, which has a within-run precision coefficient of variation $(\mathrm{CV})$ of $\leq 8 \%$ and a total $\mathrm{CV}$ of $\leq 12 \%$. Maternal vitamin $\mathrm{D}$ level was not assayed. We defined vitamin D status of the infants according to Munns et al. ${ }^{4}-25(\mathrm{OH}) \mathrm{D}$ $>50 \mathrm{nmol} / \mathrm{L}$ as sufficient, $25(\mathrm{OH}) \mathrm{D} 30.0-50.0 \mathrm{nmol} / \mathrm{L}$ as insufficient, and $25(\mathrm{OH}) \mathrm{D}<30.0 \mathrm{nmol} / \mathrm{L}$ as deficient.

T-test and Mann-Whitney U test were used to compare the mean quantitative variables while the differences between mean qualitative variables were analysed with Pearson's chi-square or Fisher's exact test. A $P$ value of $<0.05$ was considered statistically significant. For the ease of statistical analysis, infants with vitamin D insufficiency and sufficiency (non-deficient) were grouped together.

Of the 110 term neonates (mean age: $2.5[ \pm 0.9]$ weeks; $60.0 \%$ males; Malay $70.0 \%$, Chinese $20.9 \%$, Indian $8.2 \%$, other ethnicities $0.9 \%), 79.1 \%(n=87)$ were exclusively breastfed, including $80.5 \%(62 / 77)$ of the Malays and $75.8 \%$ (25/33) of the non-Malays $(P=0.574)$. None of the 110 neonates had a serum vitamin D level of $<20 \mathrm{nmol} / \mathrm{L} ; 47(42.7 \%)$ were $20.1-30.0 \mathrm{nmol} / \mathrm{L}, 33(30.0 \%) 30.1-40.0 \mathrm{nmol} / \mathrm{L}$, and $11(10.0 \%) 40.1-50.0 \mathrm{nmol} / \mathrm{L}$. Only $17.3 \%(\mathrm{n}=19)$ were vitamin D sufficient. The overall mean (SD) serum vitamin D level of all neonates was $36.9( \pm 15.3)$ $\mathrm{nmol} / \mathrm{L}$. No correlation between serum calcium level and degree of vitamin D deficiency was observed. None of the neonates had clinical symptoms or signs of vitamin D deficiency.

On univariate analysis, the only factor significantly associated with vitamin D deficiency was a shorter duration of sunlight exposure (mean $[ \pm \mathrm{SD}]$ duration of exposure, hour/week; deficient versus non-deficient: $2.00[ \pm 1.40]$ vs $2.60[ \pm 1.63], P=0.043)$.

Our results showing vitamin $\mathrm{D}$ deficiency and insufficiency is common in Malaysian neonates are consistent with studies from other tropical countries. ${ }^{5}$ We did not find any correlation between exclusive breastfeeding and vitamin D deficiency, possibly due to the small number of study subjects and a high exclusive breastfeeding rate in the present cohort. Other authors have observed that exclusive breastfeeding without adequate sunlight exposure and vitamin D supplementation were risk factors for vitamin D deficiency in neonates. ${ }^{6}$ It is likely that changes in lifestyle related to urbanisation, increased indoor activities and use of sunscreen have increased the prevalence of hypovitaminosis D in tropical countries, including pregnant mothers.

We found that the only significant risk factor for vitamin D deficiency was a shorter duration of sunlight exposure. A possible effective strategy to improve vitamin $\mathrm{D}$ level in the newborn is having adequate sunlight exposure. A study from India showed 
Table 1. Factors associated with vitamin D deficiency in 110 Malaysian neonates

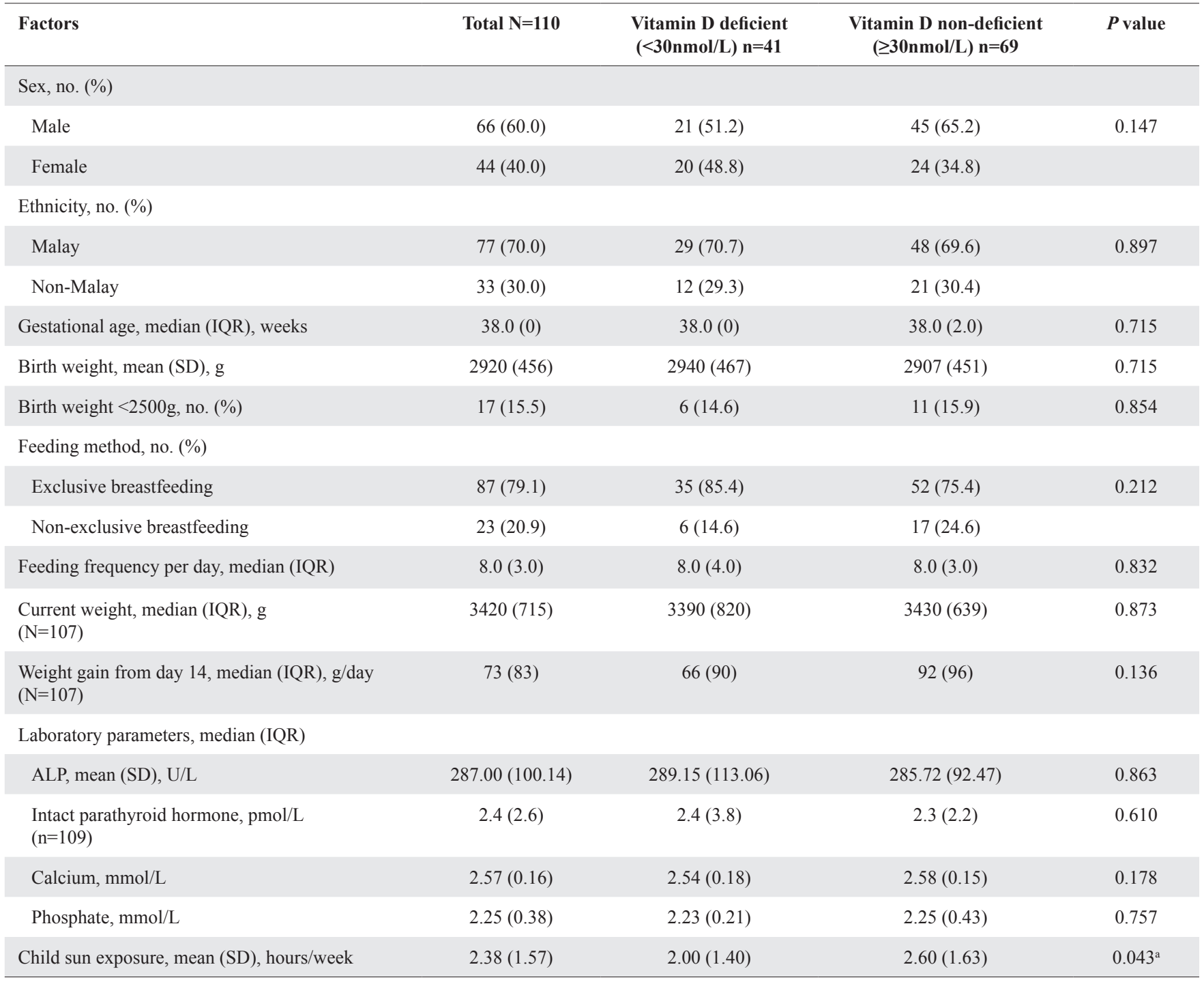

ALP: alkaline phosphatase; IQR: interquartile range; SD: standard deviation a $P<0.05$

that a minimum of 30 minutes weekly sunlight exposure in the afternoon between $10 \mathrm{am}$ and $3 \mathrm{pm}$, over $40 \%$ of the body area with the infant clothed in diapers in prone position for at least 16 weeks, was all that was required to achieve sufficient vitamin D levels by 6 months of age. ${ }^{7}$ In the present study, the mean duration of sunlight exposure for the whole cohort was 2.38 hours/week, but the 19 neonates who had sufficient vitamin $\mathrm{D}(>50 \mathrm{nmol} / \mathrm{L})$ had a mean duration of 3.21 (1.39) hours/week of sunlight exposure. Unfortunately data on the body area and the timing of day the neonates were exposed to sunlight were unavailable.

Other possible strategies to prevent neonatal vitamin D deficiency include supplementing high-dose vitamin D $(4,000$ or $6,400 \mathrm{IU} /$ day $)$ to lactating mothers with limited sunlight exposure, ${ }^{8}$ or supplementing vitamin D to all newborn babies irrespective of their breastfeeding status. Selective supplementation of vitamin D to breastfed infants, ${ }^{9}$ however, should be avoided as it is associated with concerns that lactating mothers would switch to infant formula due to the potential negative perception with breastfeeding. ${ }^{10}$

Drawbacks of the present study include a lack of maternal vitamin D level and the small sample size. Data on breastfeeding jaundice, caused by inadequate breastmilk leading to inadequate vitamin $\mathrm{D}$ intake in the first few days of life, were also not collected. Finally, data on phototherapy for neonatal jaundice, which may increase the level of vitamin $\mathrm{D}$ in babies with low levels of vitamin $\mathrm{D}$, were unavailable. 
In summary, in this cohort of Malaysian neonates, we found a very high prevalence of vitamin D deficiency and insufficiency. Supplementing pregnant and lactating mothers, and all newborn infants with vitamin D as well as ensuring adequate sunlight exposure a few months after birth are possible strategies to prevent neonatal vitamin D deficiency.

\section{Acknowledgements}

The authors would like to thank the paediatric and nursing staff of the Children's Clinic, University Malaya Medical Centre.

\section{REFERENCES}

1. Holick MF. Vitamin D deficiency. N Engl J Med 2007;357:266-81.

2. Mohamed HJJ, Rowan A, Fong B, et al. Maternal serum and breast milk vitamin D levels: findings from the Universiti Sains Malaysia Pregnancy Cohort Study. PLoS One 2014;9:e100705.

3. Barger-Lux MJ, Heaney RP. Effects of above average summer sun exposure on serum 25-hydroxyvitamin D and calcium absorption. J Clin Endocrinol Metab 2002;87:4952-6.

4. Munns CF, Shaw N, Kiely M, et al. Global consensus recommendations on prevention and management of nutritional rickets. Horm Res Paediatr 2016;85:83-106.

5. Palacios C, Gonzalez L. Is vitamin D deficiency a major global public health problem? J Steroid Biochem Mol Biol 2014; 144:138-45.

6. Dawodu A, Salameh KM, Al-Janahi NS, et al. The effect of high-dose postpartum maternal vitamin D supplementation alone compared with maternal plus infant vitamin D supplementation in breastfeeding infants in a high-risk population. A randomized controlled trial. Nutrients 2019;11:1632.

7. Meena P, Dabas A, Shah D, et al. Sunlight exposure and vitamin D status in breastfed infants. Indian Pediatr 2017;54:105-11.

8. Wagner CL, Hulsey TC, Fanning D, et al. High-dose vitamin D3 supplementation in a cohort of breastfeeding mothers and their infants: a 6-month follow-up pilot study. Breastfeed Med 2006; $1: 59-70$.

9. Wagner CL, Greer FR, American Academy of Pediatrics Section on Breastfeeding, et al. Prevention of rickets and vitamin D deficiency in infants, children, and adolescents. Pediatrics 2008;122:1142-52.

10. Perrine CG, Sharma AJ, Jefferds MED, et al. Adherence to vitamin D recommendations among US infants. Pediatrics 2010;125: $627-32$.

Way Seah Lee ${ }^{1}{ }_{F R C P C H}$, Sean Yee Wong ${ }^{1}{ }_{M B B S \text { (Malaya), }}$ Shin Yee Wong ${ }^{1} P h D$, Zhong Ling Koay ${ }^{1}$ MBBS (Malaya), Nong Sofea Ku $\underline{\text { Safuan }}{ }^{2}$ MBBS (Malaya),

Zhi Heng $\underline{\text { Sam }}{ }^{2}$ MPaeds (Malaya),

Muhammad Yaziud Jalaludin ${ }^{1}$ MPaeds (Malaya), Choong Yi Fong ${ }^{1}{ }_{F R C P C H}$, Lucy CS Lum ${ }^{1}{ }_{M R C P(U K)}$

${ }^{1}$ Department of Paediatrics, Faculty of Medicine, University of Malaya, Malaysia

${ }^{2}$ Department of Paediatrics, University Malaya Medical Centre, Malaysia

Correspondence: Prof Way Seah Lee, Department of Paediatrics, Faculty of Medicine, University of Malaya, 59100 Kuala Lumpur, Malaysia. Email: leews@um.edu.my 